\title{
Frecuencia de parásitos gastrointestinales en burros criollos (Equus africanus asinus) en el departamento de Córdoba, Colombia
}

\author{
Frequency of gastrointestinal parasites in creole donkeys (Equus africanus \\ asinus) in the department of Cordoba, Colombia
}

\author{
HERRERA B. YONAIRO ${ }^{*}$ MVZ, VERGARAA. JHEREMMYS ${ }^{2}$ MVZ, \\ ENSUNCHO H. CARLOS ${ }^{1}$ MVZ, CAUSIL V. LUIS ${ }^{1}$ Biol.
}

\begin{abstract}
${ }^{1}$ Universidad de Córdoba. Programa de Medicina Veterinaria y Zootecnia. Departamento de Ciencias Pecuarias. Montería. Colombia. 2Universidad de Córdoba. Programa de Medicina Veterinaria y Zootecnia. Departamento de Ciencias Pecuarias. Grupo de Investigación en Medicina de Grandes Animales. - MEGA. Montería. Colombia.
\end{abstract}

\section{Keywords:}

Nematodes, cestodes, Protozoa, Trichostrongylus.

\section{Abstract}

The present study was designed with the objective of determining the frequency of gastrointestinal parasites in Creole burros (donkey) in the department of Cordoba, Colombia. The type of study was cross-sectional descriptive. The study was conducted on 47 farms between August and November 2015. coprological samples were collected and flotation technique was used, examined 144 samples of the 144 samples examined $136(94.44 \%)$ were positive least for some kind of parasite. The frequencies were found Trichostrongylus spp. 91.67\% Strongylus spp. $68.06 \%$, $66.67 \%$ westeri Strongyloides, Parascaris equorum $9.72 \%, 5.56 \%$ and Anoplocephala perfoliata Eimeria spp. $2.78 \%$. It was concluded that there is a high presence of nematodes compared with tapeworms and protozoa found in the asses of Córdoba department, Colombia.

\section{Resumen}

El presente estudio fue diseñado con el objetivo de determinar la frecuencia de parásitos gastrointestinales en burros criollos (Equus africanus asinus) del departamento de Córdoba, Colombia. El tipo de estudio fue descriptivo transversal. El estudio se llevó a cabo en 47 predios entre los meses de agosto y noviembre de 2015 . Se recolectaron muestras coprológicas y se empleó la técnica de flotación, examinándose 144 muestras, De las 144 muestras examinadas $136(94,44 \%)$ resultaron positivas al menos para algún género de parásito. Las frecuencias encontradas fueron de Trichostrongylus spp. 91,67\%, Strongylus spp. 68.06\%, Strongyloides westeri 66,67\%, Parascaris equorum 9,72\%, Anoplocephala perfoliata $5,56 \%$ y Eimeria spp. $2,78 \%$. Se pudo concluir que existe una elevada presencia de nematodos en comparación con los cestodos y protozoarios, encontrados en los asnos del departamento de Córdoba, Colombia. 


\section{Introducción}

A pesar del aumento en la mecanización a nivel mundial, los burros todavía tienen el título de "bestias de carga". Ellos tienen un lugar destacado en el sistema agrícola de muchos países en desarrollo. El bajo nivel de desarrollo de las carreteras en algunas regiones y terrenos difíciles en otras partes hacen del burro una especie valiosa, adecuada y asequible como animal de carga, especialmente en los sistemas de agricultura a pequeña escala. Millones de personas en el mundo dependen de estos animales como medio de transporte (HOSSEINI et al., 2009).

Aunque el burro a menudo ha sido descrito como un animal robusto, puede padecer una variedad de enfermedades. HOSSEINI et al (2009), mencionan que los équidos han servido como hospedadores para más de 75 especies de helmintos. Estos parásitos causan daño a los animales, tanto en la fase de infección como en las etapas larvales, estas se desarrollan y surgen los parásitos adultos (WANNAS et al., 2012). Entre los helmintos, los nematodos "estróngilos" son los habitantes más comunes del tracto gastrointestinal de los equinos (WAQAS et al., 2014).

Existen pocos trabajos sobre las especies parasitarias en asnos y muy pocas publicaciones muestran estudios sobre la prevalencia de los parásitos internos de estos animales en Colombia, mientras que en países como Irán, Brasil, Etiopia si tienen reportes (MATTHEEA et al., 2002, WAQAS et al., 2014, HOSSEINI et al., 2009). Esto ha llevado a extrapolar la información que se conoce de los caballos para crear suposiciones acerca de la epidemiología y la patogenicidad de varias especies de helmintos en los burros (WAQAS et al., 2014). Un estudio desarrollado con el objetivo de determinar la asociación entre la pobreza y las enfermedades de los animales, se identificó que el parasitismo es uno de los problemas más importantes para los équidos en los países en desarrollo (SELEBAFMAYANI y GAREDAGHI, 2015).

Los estudios sobre el valor de las prácticas de control estratégico de helmintos en burros son limitados y se han basado sólo en los datos de recuento de huevos fecales (MATTHEEA et al., 2002). En vista de la considerable población de asnos en el departamento, su importancia socioeconómica y la falta de información acerca de estos helmintos, el presente estudio fue diseñado para determinar la frecuencia de parásitos gastrointestinales en burros criollos del departamento de Córdoba, Colombia.

\section{Materiales y método}

Área de estudio: El estudio se llevó a cabo en seis municipios ubicados en el departamento de Córdoba, Colombia, ubicado entre las coordenadas $7^{\circ} 23^{\prime}$ y $9^{\circ} 26^{\prime}$ de latitud norte y los $74^{\circ} 52^{\prime}$ y $76^{\circ} 32^{\prime}$ de longitud oeste, a una altura de $30 \mathrm{msnm}$, con temperatura promedio anual de $28^{\circ} \mathrm{C}$, humedad relativa del $84 \%$, precipitación media anual de $1200 \mathrm{~mm}$ y pertenece a la formación climática de bosque tropical lluvioso (CASSAB et al., 2010; PABÓN et al., 2001).

Animales de estudio: Para esta investigación se trabajó con burros criollos ( $E$. africanus asinus) con el fin de determinar la frecuencia de parásitos gastrointestinales en estos animales. Los animales fueron clasificados por sexo, 64 hembras y 80 machos. El estudio fue de tipo descriptivo transversal y se llevó a cabo entre agosto y noviembre de 2015. El tamaño de la muestra fue no probabilístico por conveniencia y se procesaron 144 muestras de heces.

Métodos parasitológicos: Las muestras se recolectaron directamente del recto del animal y de heces recién defecadas. Luego fueron depositadas en frascos estériles recolectores de materia fecal de $5 \times 6 \mathrm{~cm}$, debidamente rotulados con el sexo del animal. Las muestras recolectadas fueron almacenadas en cavas de icopor ${ }^{\circledR}$ con bolsas de geles congelados, evitando al máximo la exposición a factores externos como calor y corrientes de aire que pudieran ocasionar daños a las muestras, las cuales se transportaron al laboratorio de Parasitología de la Facultad de Medicina Veterinaria y Zootecnia de la Universidad de Córdoba, en un tiempo menor a doce horas, donde se procedió al análisis. Las muestras se examinaron por las técnicas de flotación siguiendo los procedimientos estándar para helmintos, para ello se tomaron 2 gramos de heces y me 
mezclaron con una solución sobresaturada de azúcar, posteriormente se filtró con gasa y la solución liquida fue llevada a tubos de ensayo de $15 \mathrm{ml}$ hasta la formación de un menisco convexo para colocar un cubre objetos durante 15 minutos, finalmente se retiró el cubreobjetos y se colocó sobre una lámina portaobjetos y se observó con microscopio óptico con objetivos de 10x y 40x (HENDRIX y ROBINSON, 2014). La identificación de los huevos se hizo con base a su morfología (URQUHART et al., 2003). También se realizó una clasificación de acuerdo al tipo de asociación parasitaria.

Análisis estadístico: Los resultados de esta investigación se tabularon en Microsoft Excel versión 2010 y analizados por el programa estadístico InfoStat e2015, estimando la frecuencia de cada parasito mediante la fórmula descrita por ALTMAN y BLAND (2001): Frecuencia $=($ No. Animales positivos / Población total) x 100 .

\section{Resultados}

De acuerdo a los resultados obtenidos, de las 144 muestras examinadas, el 94,44\% (136) resultó positivo al menos para algún grupo parasitario. En los burros de las fincas objeto de estudio se observó la constante presencia de huevos de helmintos, siendo los nematodos el grupo parasitario con mayores frecuencias, siendo los helmintos nematodos más frecuentes Trichostrongylus axei (91,67\%), nematodos de la familia Strongylidae $(68,06 \%)$ y Strongyloides westeri $(66,67 \%)$. Los parásitos gastrointestinales encontrados en el presente estudio se pueden observar en la Tabla 1, evidenciando la presencia de 6 géneros de parásitos en el Burro Criollo Colombiano.

En la Tabla 2, se observan la frecuencia parasitaria de acuerdo al sexo de los animales. De las 64 hembras muestreadas, 60 resultaron positivas a por lo menos un grupo parasitario, mientras que 76 machos arrojaron resultado positivo en la prueba diagnóstica.

En la Tabla 3, se detalla la clasificación de acuerdo a las asociaciones parasitarias.
Tabla 1: Frecuencia por género parasitario en burro criollo colombiano.

\begin{tabular}{|c|c|c|c|}
\hline Genero & Positivos & $\begin{array}{c}\text { Frecuencia } \\
(\%)\end{array}$ & $\begin{array}{c}\text { Clase } \\
\text { parasitaria }\end{array}$ \\
\hline Trichostrongylus spp. & 132 & 91,67 & Nematodo \\
\hline Strongylidos spp. & 98 & 68,06 & Nematodo \\
\hline Strongyloides westeri. & 96 & 66,67 & Nematodo \\
\hline Parascaris equorum & 14 & 9,72 & Nematodo \\
\hline $\begin{array}{l}\text { Anoplocephala } \\
\text { perfoliata }\end{array}$ & 8 & 5,56 & Cestodo \\
\hline Eimeria spp. & 4 & 2,78 & Protozooario \\
\hline
\end{tabular}

Tabla 2: Frecuencia por sexo de helmintos gastrointestinales en burro criollo Colombiano

\begin{tabular}{ccc}
\hline \multirow{2}{*}{ Parásito } & Machos (\%) & Hembras (\%) \\
\cline { 2 - 3 } & \multicolumn{2}{c}{ Positivos } \\
\hline Trichostrongylus spp. & 92,5 & 90,6 \\
Strongylidos spp. & 65 & 71,8 \\
Strongyloides westeri. & 67,5 & 65,6 \\
Parascaris equorum & 10 & 9,3 \\
Anoplocephala perfoliata & 7,5 & 3,1 \\
\hline
\end{tabular}

Tabla 3. Asociaciones parasitarias en burro criollo colombiano

\begin{tabular}{ccc}
\hline Taxones & $\begin{array}{c}\text { Burros } \\
\text { parasitados }\end{array}$ & $\%^{1}$ \\
\hline Monoparasitismo & 8 & 5,88 \\
Biparasitismo & 48 & 35,29 \\
Triparasitismo & 72 & 52,94 \\
Tetraparasitismo & 8 & 5,88 \\
\hline
\end{tabular}

${ }^{1}$ En base a 136 muestras positivas 
En la Figura 1 se pueden observar algunos de los huevos de parásitos encontrados en el estudio.
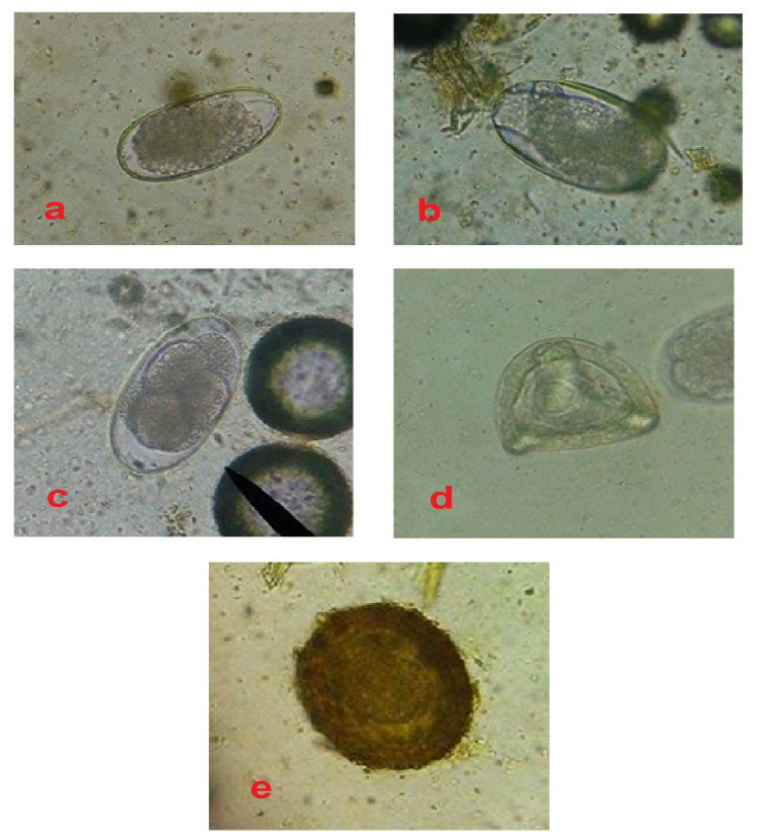

Figura 1: Huevos de parásitos gastrointestinales en Burros Criollos. a: huevo tipo Trichostrongylus spp., b: huevo de S. westeri, c: huevo tipo Strongylido, d: huevo de $A$. perfoliata, e: huevo de $P$. equorum

\section{Discusión}

Entre los helmintos de los équidos, los parásitos del tracto intestinal son los más conocidos y comunes. Los grandes y pequeños Stróngylus, son los parásitos frecuentes del intestino grueso, cuyas larvas migran de manera compleja a todo el organismo y son responsables de problemas variados y a menudo graves en los equinos; y Parascaris equorum (particularmente en animales jóvenes) (MORALES et al., 2010; PRADA y ROMERO 2009). Por ello, es de vital importancia cuando se trabaja con una población equina, conocer qué poblaciones de parásitos están presentes, ya que muchos pueden ser poco o nada patógenos, mientras que otras poblaciones pueden ser altamente patógenas, llevando incluso a la muerte de los huéspedes. De igual forma, conocer la población parasitaria presente, permite elegir el antiparasitario específico contra los parásitos identificados (PRADA y ROMERO 2009).
El presente estudio determinó una frecuencia de $94,44 \%$ (136/144) de parásitos gastrointestinales en burros criollos en el departamento de Córdoba. Estos resultados son considerados similares a los encontrados por GATACHEW et al. (2010) en el Reino Unido, IBRAHIM et al. (2001) en Etiopia y BURDEN et al. (2010) en México, donde las prevalencias fueron de $99,96,9$ y $82 \%$ respectivamente.

En Colombia son pocos los estudios reportados sobre frecuencia y prevalencia de parásitos gastrointestinales que afectan a los burros criollos (E. asinus). El único estudio realizado en esta especie en el país fue el realizado por RIOS et al (2011), en el municipio de Oiba (Santander). Es de anotar que en ese estudio solo se muestrearon 16 asnales, encontrando prevalencias para los équidos en general de $90 \%$ similares a los hallados en esta investigación.

La alta frecuencia de helmintos nematodos en el estudio podría deberse al manejo dado a estos animales en el departamento de Córdoba, en donde prácticamente no existen planes sanitarios para esta especie, siendo este considerado un équido de trabajo y de tracción animal. Al respecto ALUJA et al. (2000) y UPJHON et al. (2010), consideran que en los países en vías de desarrollo la mayoría de los équidos de trabajo y en especial los dedicados a la tracción son propiedad de personas con bajos recursos económicos, cuyo sustento se deriva de la actividad diaria de estos animales, situación que conduce a un manejo inadecuado, asociado a largas jornadas de trabajo, insuficiente nutrición y deficiente atención sanitaria, hecho que favorece la exposición y susceptibilidad a distintos agentes infecciosos, incluyendo los parásitos (CASTAÑO 2005; MORALES et al., 2012; CUTOLO et al., 2011).

En todo el mundo los équidos (cebra, burro, caballo y sus híbridos) experimentan algún nivel de parasitismo de forma continua, siendo los équidos de trabajo en los países en vías de desarrollo los más susceptibles a presentar altas cargas parasitarias (CRANE et al., 2011; UPJOHN et al., 2010).

Estos datos son superiores y diferentes a los reportados por WAQAS et al. (2014) en Pakistán, donde los géneros más prevalentes 
fueron Strongylus $(28,33 \%)$ y Trichostrongylus $(9,66 \%)$ y WANNAS et al. (2012) en Iraq donde las prevalencias por genero fueron Strongylidae $(57,14 \%)$, P. equorum $(32,14 \%)$, S. westeri (28,57\%), Trichostrongylus axei $(17,85 \%)$. Esta diferencia en cuanto al grado de infestación pudo haber sido influenciada por el manejo sanitario que se les dan a los animales en las diferentes regiones donde se realizaron los estudios. A diferencia de los otros países en mención, en nuestra región no se les presta la atención clínica necesaria a los burros y están en manos de pequeños productores y campesinos que en la mayoría de los casos no manejan un plan de desparasitación adecuado.

En el estudio cabe resaltar la ausencia de huevos de Oxyuris equi y la presencia de huevos de helmintos cestodos de $A$. perfoliata (5,56\%); en comparación a las otras investigaciones que si lo reportan Oxyuris equi y la no presencia de $A$. perfoliata.

La frecuencia de los parásitos encontrados es diferente en distintas partes del mundo y pueden ser debido a las variaciones en las condiciones climáticas y a los distintos programas de prevención y control frente a los helmintos. MATTHEEA et al. (2002) considera que la temperatura, precipitación, humedad relativa, distribución geográfica y las condiciones bajo las cuales los animales son mantenidos son factores que interfieren en la prevalencia de los parásitos.

GÜIRIS (2010) y VALDÉZ (2013) mencionan que el clima de la región juega un papel importante en las especies de helmintos y su abundancia, se evidencia que estos parásitos son comunes en équidos de regiones tropicales como México, Panamá y Brasil.

En relación al sexo, en el estudio no se encontraron diferencias en relación a los géneros parasitarios, siendo las infecciones similares tanto en machos como en hembras. Esto quizás sea debido a que las condiciones de pastoreo y manejo que presentan los animales en el departamento son los mismos para ambos sexos. MUGHINI et al. (2011) reporta un estudio en Italia donde demuestra que el sexo no es relevante con respecto a las cargas parasitarias. En contraste MBAFOR et al. (2012) y FRANCISCO et al. (2009) demostraron en équidos mayores prevalencias de estróngilos que en hembras.

En el estudio, la mayoría de los animales presentan infecciones mixtas, presentando 128 burros $(94,11 \%)$ multiparasitismo, siendo el bi y triparasitismo las asociaciones más frecuentes con 35,29 y $52,94 \%$ respectivamente. Este tipo de asociaciones son frecuentes en los animales domésticos en pastoreo, puesto que las condiciones ambientales en los países tropicales facilitan a los helmintos nematodos su gran capacidad de reproducción y adaptación a diferentes condiciones climáticas (SOCA et al, 2007).

Es de anotar que es importante determinar los géneros de parásitos más frecuentes, ya que algunas pueden ser poco o nada patógenas, mientras que otras pueden serlo altamente, llevando incluso a la muerte de los hospederos (PRADA y ROMERO 2009). En el estudio el género predominante fue Trichostrongylus spp., parasito que se alimenta de tejidos de la mucosa gastrointestinal y está asociado a procesos diarreicos y retardo en el crecimiento. La alta frecuencia encontrada de este nematodo quizás este dada por la capacidad que tiene este, de resistir las altas temperaturas (VÁSQUEZ et al. 2004). Además, FINCHER y STEWART (1979) en estudio en nematodos aseguran que el suelo es un reservorio importante para larvas infectantes las cuales en condiciones adversas pueden migrar a las pasturas. LEVINE (1968) lo cita como el único parasito común entre equinos y bovinos. Además, la alta frecuencia también puede atribuirse porque en el departamento de Córdoba es común el pastoreo conjunto de rumiantes y équidos en los sistemas de producción.

Los signos clínicos asociados a parasitismos intensos incluyen: reducción de la tasa de crecimiento, distensión abdominal, cólico por compactación, mala condición corporal, debilidad, disminución del rendimiento físico y de la fertilidad; tasa de digestión y absorción de nutrientes reducida; perforación intestinal y muerte, entre otros (CUTOLO et al., 2011). Si bien en el estudio se encontraron altas frecuencias en burros, la carga parasitaria por animal indicaría que su importancia patológica sería de poca magnitud, ya que los animales no presentaban signos 
propios del parasitismo gastrointestinal. Sin embargo, la presencia en la especie animal tiene la trascendencia de mantenerlos como diseminadores y reservorios de la infección para otros animales más susceptibles.

En general, en Colombia, como en muchas partes del mundo, la atención dada por los propietarios a los burros es muy deficiente y está por debajo de los planes sanitarios implementados en otras especies. Esto podría deberse en parte a la percepción errónea que se tiene acerca de la crianza de los burros, en donde se considera que estos animales requieren poca atención, condicionando de esta manera la alta frecuencia del parasitismo gastrointestinal y las infecciones mixtas. Factores como la alta contaminación larval en los pastizales y la intensidad de pastoreo puede aumentar la probabilidad de ingestión de huevos y larvas y con ello favorecer la infección y el multiparasitismo.

\section{Conclusiones}

Existe una alta frecuencia de infección parasitaria en los hatos de burros estudiados en el departamento de Córdoba. Las infecciones por helmintos nematodos pueden considerarse altas, lo que sugiere que el parasitismo gastrointestinal es un problema de importancia médica que deber ser objeto de mayor atención para su prevención y control.

Los principales helmintos hallados fueron nematodos del genero Trichostrongylus spp, estróngilos y $S$. westeri, pudiéndose considerar a estos parásitos como endémicos en la región. La presencia de estos helmintos obliga a establecer planes sanitarios de desparasitación que regulen los ciclos parasitarios y minimicen el impacto de estas parasitosis en el estado de salud y rendimiento económico y productivo de estos animales.

\section{Referencias}

ALTMAN, D.G.; BLAND, J.M. 2001. Statistics Notes: Diagnostic tests 1: sensitivity and specificity. Brit. Med. J. 308:1552-1553.

ALUJA, A.; LOPEZ, A.; CHAVIRA, H.; OSEGUERA, D. 2000. Condiciones patológicas más frecuentes en los équidos de trabajo en el campo mexicano. Revista Veterinaria Mexico 31(2):65 - 168.

BURDEN, F. et al. 2010. Selected health and management issues facing working donkeys presented for veterinary treatment in rural Mexico: Rev. Zooc. 2014. 1(1):14-23

CASSAB, A.; MORALES, V.; MATTAR, S. 2010. Factores climáticos y casos de Dengue en Montería, Colombia. 2003-2008. Rev. Salud pública 13 (1):115-128.

CASTAÑO, R. 2005. Parásitos de los equinos. Red de Helmintologia Para America Latina y El Caribe. FAO- INTA, Argentina.

CRANE, M.; KHALLAAYOUNE, K.; SCLANTEBURY, C.; CHRISTLEY, R. 2011. A randomized triple blind trial to assess the effect of an anthelmintic programme for working equids in Morocco. BMC Veterinary Research 7 (1):1-8.

CUTOLO, A.; TINTINO, A.; MAEQUES, S. 2011. Field study on the efficacy of an oral $2 \%$ ivermectin formulation in horses. Revis Brasilera De Parasitologia Veterinaria 20 (2):171-175.

FINCHER, T.; STEWART, B. 1979. Vertical Migration by Nematode Larvae of Cattle Parasites Through Soil. Proc. Helm. Soc. Wash. 46 (1):43-46. 
FRANCISCO, I.; ARIAS, M.; CORTIÑAS, F.J.; FRANCISCO, R.; MOCHALES, E.; SÁNCHEZ, J.A, et al. 2009. Silvopastoralism and autochthonous equine livestock: Analysis of the infection by endoparasites. Vet Parasitol 14164 (2-4):357-62.

GATACHEW, M.; TRAWFORD, A.; FESEHA, G.; REID, S. 2010. Gastrointestinal parasites of working donkeys of Ethiopia. Tropical Animal Health and Production 41(2):27-33.

GÜIRIS, A. et al. 2010. Biodiversity and distribution of helminths and protozoa in naturally infected horses from the biosphere reserve "La Sierra Madre de Chiapas", México. Veterinary Parasitology 164:357-362.

HENDRIX, C.M.; ROBINSON, E.D. 2014. Diagnostic parasitology for veterinary technicians. Elsevier Health Sciences. USA.

HOSSEINI, S.H.; MESHGI, B.; ESLAMI, A.; BOKAI, S.; SOBHANI, M.; EBRAHIMI SAMANI R. 2009. Prevalence and biodiversity of helminth parasites in donkeys (Equus assinus) in Iran. Int. J. Vet. Res. $3(2): 95-99$.

IBRAHIM, N.; BERHANU, T.; DERESSA, B.; TADELE, T. 2001. Survey of Prevalence of Helminth Parasites of Donkeys in and Around Hawassa Town, Southern Ethiopia. Global Veterinaria 6 (3):223-227.

LEVINE, N.D. 1968. Nematodes parasites of domestic animals and of man. Burgues Publishing Co. Minnesota, USA.

MATTHEEA, S.; KRECEKA, R.C.; MILNEA, S.A.; BOSHOFFA, M.; GUTHRIEB, AJ. 2002. Impact of management interventions on helminth levels, and body and blood measurements in working donkeys in South Africa. Veterinary Parasitology 107:103-113.

MBAFOR, F.; KHAN, V.; JOSUEQW; TCHOUMBOUE, J. 2012. Prevalence and intensity of gastro-intestinal helminths in horses in the Sudano-Guinean climatic zone of Cameroon. Tropical Parasitoligy 2 (1):45-48.

MORALES, A.; BELLO, H.; GOMEZ, M. 2012. Prevalencia de parásitos gastrointestinales en caballos pura sangre de carrera (Equus Caballus) durante el periodo de cuarentena 2010 en el Hipodromo "La Rinconada" Caracas, Venezuela. Neotrop. Helmintho 6 (1):115-119.

MORALES, A.A.; GARCÍA, F.; CORONADO, T.R.; LATOUCHE, O.; RIVERO, L.; ROSSINI, M.; BELLO, H.; LEAL, L.; LÓPEZ, P.; RODRÍGUEZ, C.F. 2010. Síndrome de enteritis secretora crónica parasitaria por Strongylus vulgaris con resistencia a ivermectina en un equino pura sangre de carrera. Neotropical Helminthology 4 (1):71-74.

MUGHINI, G.; USAI, F.; STANCAMPIANO, L. 2011. Strongylosis in horses slaughtered in Italy for meat production: Epidemiology, influence of the horse origin and evidence of parasite self-regulation. Veterinary Parasitology 179:167-174.

PABÓN, J. ESLAVA, J. GÓMEZ, R. 2001. Generalidades de la distribución espacial y temporal de la temperatura del aire y de la precipitación en Colombia. Meteorología Colombiana (4):47-59.

PRADA, A. ROMERO, C. S. 2009. Determinación de géneros de endoparásitos que afectan a los equinos de las sabanas del Casanare. Revista de Medicina Veterinaria 18:71-79.

RIOS, M.A.B.; QUICENO, V.H.A.; ARIAS, D.A.D.; PLATA, E.A.R. 2011. Prevalencia de parásitos gastrointestinales en équidos del municipio de Oiba (Santander). Spei Domus 7 (15):2-12. 
SELEBAFMAYANI, A.; GAREDAGHI Y. 2015. Survey on Fecal Gastrointestinal Parasitic Helminthes in Horses of Jokey clubs in Ardabil city, Iran. Biological Forum - An International Journal 7 (1):106-110.

SOCA, M.; SIMÓN, L.; ROQUE, E. (2007). Árboles y nemátodos gastrointestinales en bovinos jóvenes: Un nuevo enfoque de las investigaciones. Pastos y Forrajes 30:21-33.

UPJOHN, M.M.; SHIPTON, K.; LEROTHOLI, T.; ATTWOOD, G.; VERHEYEN. K.L. 2010. Coprological prevalence and intensity of helminth infection in working horses in Lesotho. Trop Anim Health Prod 42 (8):1655-61

URQUHART, G.M.; ARMOUR, A.M.; DUNN. JENNINGS, F.W. 2003. Veterinary parasitology.3 ed. pp: 4-78.

VALDÉZ, M.; HERNANDEZ, M.; RODRIGUEZ, L.; ALONSO, M. 2013. Gastrointestinal nematode burden in working equids from humid tropical areas of central Veracruz, Mexico, and its relationship with body condition and haematological values. Trop Anim Health Prod 45:603-607.

VÁSQUEZ, V.; FLORES, J.; VALENCIA, C.; HERRERA, D.; PALACIOS, A.; LIÉBANO.; PELCASTRE, A. (2004). Frecuencia de nemátodos gastroentéricos en bovinos de tres áreas de clima subtropical húmedo de México. Técnica pecuaria en México 42 (2):237-245.

WANNAS, H.; DAWOOD, K.H.; GASSEM, G.H.; 2012. Prevalence of Gastro-intestinal Parasites in Horses and Donkeys in Al Diwaniyah Governorate AL-Qadisiya Journal of Vet.Med.Sci. 11(1):24-32.

WAQAS, M.; SARWAR KHAN, M.; ZAMEER DURRANI, A.; ARIF KHAN, M.; D AVAIS, M.; AKBAR KHAN, S.; UR REHMAN, S.; HUSSAIN, A.; NASIR, N.; HUSSAIN, A., CEZAR DOS SANTOS, F. 2014. Prevalence of Gastrointestinal parasites, chemotherapy and haematology of Strongylosis in Donkeys of District Lahore, Pakistan. Int.J.Curr.Microbiol.App.Sci. 3 (7):198-207. 